\title{
Fast Modelling of Heart Pathology with Soft Objects
}

\author{
Burkhard Wünsche $^{\mathrm{a}}$, Bo Li ${ }^{\mathrm{a}}$, Alistair A. Young ${ }^{\mathrm{b}}$ \\ ${ }^{a}$ Department of Computer Science, University of Auckland, Private Bag 92019, Auckland, New \\ Zealand \\ ${ }^{\mathrm{b}}$ Department of Anatomy with Radiology, Department of Physiology, University of Auckland, \\ Private Bag 92019, Auckland, New Zealand
}

\begin{abstract}
Heart diseases cause considerable morbidity and the prognosis after heart failure is poor. Modelling the heart pathology is useful for diagnosis, analysis, and treatment planning of cardiac diseases. This is in particular the case for congenital abnormalities where surgery is often necessary directly after birth. Over the past decade, a variety of tools for ventricular modelling have been suggested. However, most of them require either substantial user interaction or make use of the default topology of a healthy heart. In this paper we present a modelling tool based on soft objects which allows a fast semi-automatic approximation of the heart pathology. The tool requires little user interaction, works well for normal and abnormal heart topologies, and makes it easy to model cardiac arteries and veins.
\end{abstract}

Key words: Ventricular modelling, heart modelling, soft objects, heart pathology, isosurfaces

Email addresses: burkhard@cs.auckland.ac.nz (Burkhard Wünsche), B.li@auckland.ac.nz (Bo Li), a.young@auckland.ac.nz (Alistair A. Young).

URLs: http://www.cs.auckland.ac.nz/ burkhard (Burkhard Wünsche), http://www2 . auckland.ac.nz/anat/ayoung (Alistair A. Young). 


\section{Introduction}

Heart diseases remain the biggest killer in the western world [1]. Cardiac diseases can result in heart failure, which is a clinical syndrome that arises when the heart is unable to pump sufficient blood to meet the metabolic needs of the body at normal filling pressures [2]. The acquisition and visualization of cardiac imaging data improves the diagnosis and the understanding of the development and progress of heart diseases and is important for the planning of heart surgery. However, in many cases the display of the imaging data is not sufficient and the creation of a 3D model is necessary for additional analysis and the planning and simulation of surgical procedures. Over the past decade, a variety of manual and semi-automatic tools have been presented to model the ventricles of the heart (see for example [3,4]). However, most of these tools require considerable user interaction or use a default model of a healthy heart. As a result such tools will fail for pathological hearts with abnormal topologies. In this paper we present a modelling tool based on soft objects which allows a fast semi-automatic approximation of the heart pathology. The tool requires little user interaction, works well for normal and abnormal heart topologies, and makes it easy to model cardiac arteries and veins.

\section{Methods}

Soft object modelling (implicit surface modelling) has been originally proposed by Wyvill et al. [5] and is closely related to earlier work by Blinn [6]. Objects are represented as collections of primitives and are blended by defining a density field $f_{i}(\mathbf{x})$ for each primitive $i$. The density field equals one on the primitive and smoothly decreases to zero at a distance of $R$ (the radius of influence) from the primitive. The soft object is then defined as the 0.5-isosurface of the field obtained by summing up the field functions of all primitives, i.e. the surface of the soft object is defined as all points $\mathbf{x}$ for which $F(\mathbf{x})=\sum_{i} f_{i}(\mathbf{x})=0.5$. The original field function proposed by Wyvill et al. is [5]:

$$
D(r, R)=1-\frac{4}{9}\left(\frac{r}{R}\right)^{6}+\frac{17}{9}\left(\frac{r}{R}\right)^{4}-\frac{22}{9}\left(\frac{r}{R}\right)^{2}
$$

and the density field of a primitive is defined as

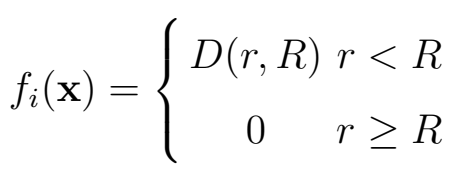


where $r=r\left(\mathbf{x}\right.$, Primitive $\left._{i}\right)$ is the distance from the point $\mathbf{x}$ to the primitive and $R=$ $R\left(\mathbf{x}\right.$, Primitive $\left._{i}\right)$ is the radius of influence of the density field of the primitive. In our case both $r$ and $R$ are dependent on the field coordinates and the type of a primitive.

We have implemented three simple primitives in order to approximate the heart's shape with soft objects:
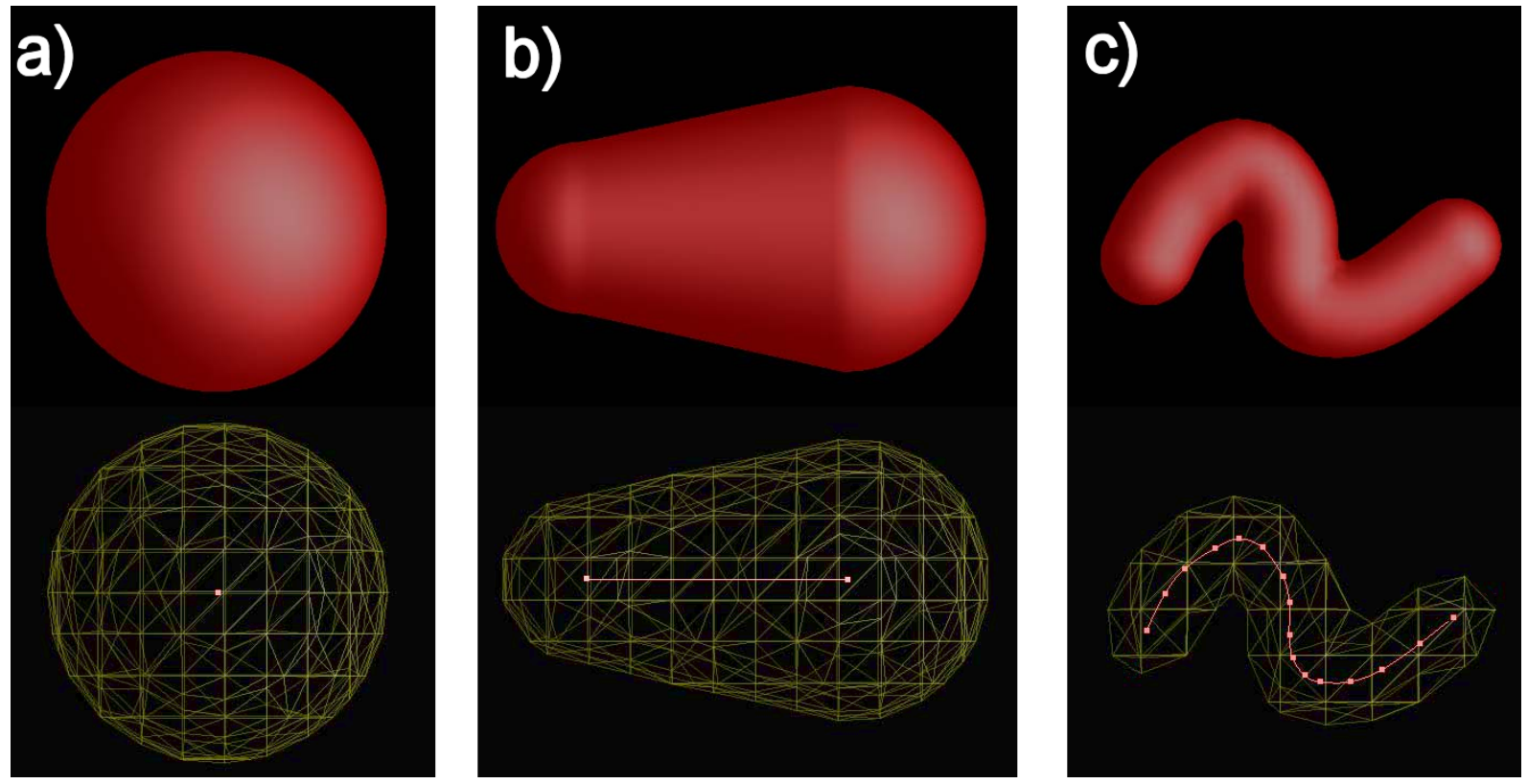

Fig. 1. Soft object primitives for cardiac modelling: (a) soft ball, (b) soft cone, (c) soft pipe.

Soft Balls are defined by a centre point $\mathbf{c}_{\text {Ball }}$ and a radius $r_{\text {Ball }}$. The resulting soft object is a sphere as illustrated in figure 1(a). The distance function for this type of primitive is the Euclidian distance to its centre point and the radius of influence of the primitive is constant.

Soft cones are defined by a line segment and one radius each for the line's start and end point. If the radii are the same the resulting soft object is a cylinder with rounded caps. If the radii are different the resulting soft object resembles a rounded cone as illustrated in figure 1(b). Soft balls and soft cones are useful for modelling the ventricular cavities and the overall shape of the heart muscle. The distance function for a soft cone is the Euclidean distance of a point to a line segment.

Finally we have implemented soft pipes which are illustrated in figure 1(c). These primitives are most useful for modelling arteries and veins connected to the heart muscle. Soft pipes are described by Catmull-Rom splines which are defined by $n$ control points. The user can define different radii for the control points of the spline which makes it possible 
to model widenings and narrowings of blood vessels. The Catmull-Rom spline has the property that all control points are interpolated and that the tangent of the curve at an interior control point is parallel to the line connecting its neighbouring control points. We tested different types of splines and decided to use Catmull-Rom splines since they have local control, they are intuitive to use, they interpolate their control points and they are piecewise cubic and hence efficient to compute. In order to compute the distance function and radius of influence we approximate the spline by a polyline and then use similar computations as for the soft cone.

Primitives can be combined by adding and subtracting density fields. In order to make our modelling tool more powerful we have added a number of additional functionalities which reflect the specific modelling problem we are trying to solve. The easiest way to define the cardiac geometry is to define the outside (epicardial) surface and inside (endocardial) surface of the heart by two separate soft objects and to subtract the inside model from the outside model. Additional modelling capabilities are obtained by allowing negative values for the radius of influence $\mathrm{R}$ of a primitive. In particular a primitive, such as soft pipe, can have both negative and positive radii at its control points.

While soft objects are easy to use and make it easy to create smooth models it is usually challenging to precisely model a particular shape. The reason for this is that when adding or subtracting fields it is difficult to predict where the isosurface of the resulting field will be. This drawback is in general not a disadvantage for our project since we are mainly interested in modelling the heart topology rather than its exact shape. However, in some cases we want to obtain an exact representation of features. An example is a "septal defect" which is a hole in the wall separating two atria or ventricles. In order to get around this limitation our toolkit allows the user to scale the density function given in equation 1 . The sharpness and diameter of the hole can be increased by increasing the scale factor. Scaling the density field can also be used when adding two density fields together. Increasing the scale factor for a primitive will increase its diameter within the resulting shape and will increase the curvature of the blending surface between the primitives.

In order to improve the quality of a user-defined model we have implemented a tool for automatic surface fitting. The steps of the process are illustrated in the four parts of figure 2. We first compute the intersection of the soft object with each MRI image plane using a two-dimensional Marching Cubes algorithm (b). We then automatically fit that contour to the closest contour on the MRI image by using B-Spline snakes (c). Finally we reconstruct the $3 \mathrm{D}$ shape of the heart by using radial basis functions (d). In order to get a smooth result we have implemented a variation which approximates the sample points on the fitted B-Spline snakes rather than interpolating them [7]. 
a)

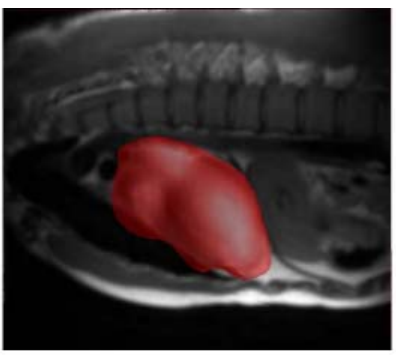

c)

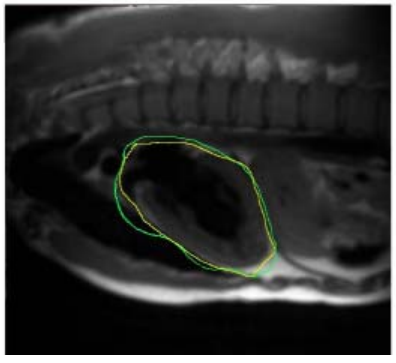

b)

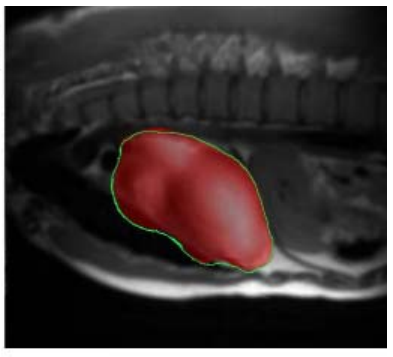

d)

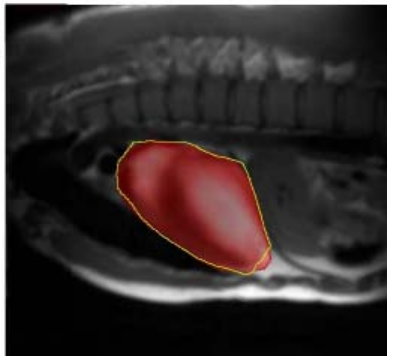

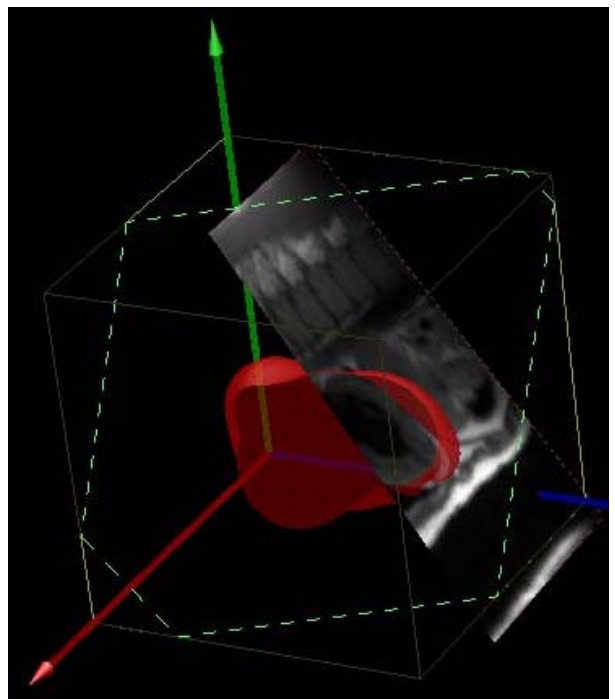

Fig. 3. The user interface allows selection and clipping of MRI image stacks.
Fig. 2. Surfaces are fitted by computing 2D contours (b), fitting them with an active contour model (c) and interpolating the contours using radial basis functions (d).

Typical heart data sets contain five stacks of MRI images. The most important image stacks are the short axis and long axis images of the heart. The other three stacks contain images parallel to the MRI coordinate system planes. Our user interface enables the user to select an image stack and to adjust the range of simultaneously displayed images using a slider. Additionally we have implemented clipping planes which can be moved along a fixed normal direction or which can be positioned arbitrarily in the 3D space by using sliders along the coordinate axes. In order to facilitate modelling the user can select whether only the MRI data set or both the data set and the soft object are clipped (see figure 3).

\section{Results}

The research described in this paper is work in progress and we have not yet performed supervised clinical trials. However, we have consulted a medical specialist and we have created various heart models and we found it relatively easy to create an approximation of the endocardial and epicardial surface of the heart. Combining the two soft objects for each surface into a single object proves more challenging due to the fact that the density field values can fluctuate considerably inside a soft object. The biggest difficulty for users which have only a limited experience with cardiac modelling is to perceive the $3 \mathrm{D}$ geometry of blood vessels connected to the heart from the MRI image data. One 
solution we might implement in future is to display the MRI data set using direct volume rendering. However, we expect that medical professionals won't report this problem since they will have considerable more experience and training with perceiving 3D anatomical structures from MRI slice data than our test users.

\section{Conclusion}

Soft objects are ideally suited to approximate smooth organ shapes such as the heart. We have introduced soft cones and soft pipes which are especially suitable for modelling blood vessels such as the ventricles and the arteries and veins. Density field scaling can be used to model important topological features such as septal defects. While clinical trials haven't been performed yet initial results are encouraging. Possible improvements include tools for modelling thin vessel walls and a user interface which improves the perception of the 3D geometry of blood vessels.

\section{References}

[1] S. Masood, G.-Z. Yang, D. J. Pennell, D. N. Firmin, Investigating intrinsic myocardial mechanics - the role of MR tagging, velocity phase mapping and diffusion imaging, Journal of Magnetic Resonance Imaging 12 (6) (2000) 873 - 883.

[2] R. W. Alexander, R. C. Schlant, V. Fuster, R. A. O'Rourke, R. Roberts, E. H. Sonnenblick (Eds.), Hurst's The Heart, $9^{t h}$ Edition, McGraw-Hill Companies, Inc., London, 1994.

[3] A. A. Young, Model tags: Direct 3D tracking of heart wall motion from tagged magnetic resonance images, Medical Image Analysis 3 (4) (1999) 361 - 372.

[4] D. Wei, Whole-heart modeling: progress, principles and applications, Progress in Biophysics and Molecular Biology 67 (1) (1997) 17 - 66.

[5] G. Wyvill, C. McPheeters, B. Wyvill, Animating soft objects, The Visual Computer 2 (4) (1986) $235-242$.

[6] J. F. Blinn, A generalization of algebraic surface drawing, ACM Transactions on Graphics 1 (3) (1982) $235-256$.

[7] J. C. Carr, R. K. Beatson, J. B. Cherrie, T. J. Mitchell, W. R. Fright, B. C. McCallum, T. R. Evans, Reconstruction and representation of 3 d objects with radial basis functions, in: Proceedings of ACM SIGGRAPH 2001, 2001, pp. $67-76$. 